\title{
Canonical formalism for the Born-Infeld particle
}

\author{
Dariusz Chruściński* \\ Fakultät für Physik, Universität Freiburg \\ Hermann-Herder-Str. 3, D-79104 Freiburg, Germany
}

\begin{abstract}
In the previous paper [1] it was shown that the nonlinear Born-Infeld field equations supplemented by the "dynamical condition" (certain boundary condition for the field along the particle's trajectory) define perfectly deterministic theory, i.e. particle's trajectory is determined without any equations of motion. In the present paper we show that this theory possesses mathematically consistent Lagrangian and Hamiltonian formulations. Moreover, it turns out that the "dynamical condition" is already present in the definition of the physical phase space and, therefore, it is a basic element in the above theory.
\end{abstract}

Freiburg THEP-98/5

*On leave from Institute of Physics, Nicholas Copernicus University, ul. Grudziądzka 5/7, 87-100 Toruń, Poland. 


\section{Introduction}

Born-Infeld electrodynamics [2] was proposed in the thierties as an alternative for Maxwell theory (see also [3] for a useful review). The main motivation of Born and Infeld was to construct a theory which has classical solutions representing electrically charged particles with finite self-energy. Born-Infeld theory indeed admits such solutions (recently Gibbons proposed [4] to call them "BIons"). However, after Dirac's paper [5] on a classical electron and the birth of quantum electrodynamics in the forties Born-Infeld theory was almost totally forgoten for a long time.

Recently, there is a new interest in this theory due to investigations in string theory. It turns out that some very natural objects in this theory, so called D-branes, are described by a kind of nonlinear Born-Infeld action (see e.g. [4] and [6]). Moreover, due to the remarkable interest in field and string theory dualities [7], the duality invariance of BornInfeld electrodynamics was studied in great details [8] (actually this invariance was already observed by Schrödinger [9]).

Our motivation to study Born-Infeld electrodynamics is not a string theory but a dynamics of a classical point charge. There are following reasons to consider nonlinear electromagnetism: it is well known that Maxwell electrodynamics when applied to pointlike objects is inconsistent (see [10] for the review). This inconsistency originates in the infinite self-energy of the point charge. In the Born-Infeld theory this self-energy is already finite. Therefore, one may hope that in the theory which gives finite value of this quantity it would be possible to describe the particle's self-interaction in a consistent way. Moreover, the assumption, that the theory is effectively nonlinear in the vicinity of the charged particle is very natural from the physical point of view and this we already learned from quantum electrodynamics (Born tried to make contact with quantum field theory by identifying Born-Infeld Lagrangian as an effective Euler-Heisenberg Lagrangian [11]. It has been shown [12] that the effective Lagrangian can coincide with those of Born and Infeld up to six-photon interaction terms only).

We consider very specific model of nonlinear theory because, among other nonlinear theories of electromagnetism, Born-Infeld theory possesses very distinguished physical properties [13]. For example it is the only causal spin-1 theory [14] (apart from the Maxwell one). Recently, Born-Infeld electrodynamics was successfully applied [15] as a model for generation of multipole moments of charged particles.

In the previous paper [1] we studied the electrodynamics of a point charge in the BornInfeld nonlinear theory. Due to the nonlinearity of the field equations it is impossible to derive separate equations of motion for the charged particle corresponding e.g. to the celebrated Lorentz-Dirac equation in the Maxwell case. Could we, therefore, determine the particle's trajectory without equations of motion? We showed [1] that it is in fact possible. Analysing the interaction between charged particle and nonlinear electromagnetism we proved that the conservation of total four-momentum of the composed (particle+field) system is equivalent to the certain boundary condition for the Born-Infeld field which has to be satisfied along the particle's trajectory. We call it "dynamical condition" (formula (11) in the present paper) because, roughly speaking, it replaces particle's equations of 
motion. Field equations supplemented by this condition define perfectly deterministic theory, i.e. initial data for the particle and field uniquely determine the evolution of the system.

In the present paper we show that the theory derived in [1] possesses consistent Lagrangian and Hamiltonian structures. It is important because any reasonable physical theory could be formulated this way. Therefore, we expect that also Born-Infeld electrodynamics completed by the dynamical condition (11) is no exception in this rule. Moreover, we claim that the mathematically well defined canonical structure is a necessary condition for the theory to be consistent. It should be stressed that our model of a charged particle is different from that of Born and Infeld, i.e. our particle is not a purely electromagnetical "BIon". Nevertheless, we call it a "Born-Infeld particle". A particle's mass, which appears e.g. in formula (10), could be interpreted as an effective mass, i.e. a "mechanical" mass completed by "radiative corrections" which are due to the electromagnetic interaction. The finite self-energy of a charge (i.e. mass of its Coulomb field) is already contained in the field energy. Due to the nonlinearity of the theory there is no way to separate this self-energy from the total field energy (in Maxwell theory this separation enables one to perform the mass renormalization [10]). It turns out that the theory with a purely electromagnetical charged particle (contrary to the one considered in the present paper) does not have any consistent Hamiltonian formulation (this observation was made long ago by Pryce [16]).

The paper is organised as follows: in the next section we briefly sketch main results of [1]. In section 3 a 2-nd order particle's Lagrangian is constructed and it is proved that the corresponding Euler-Lagrange equations are equivalent to our dynamical condition. Then in section 4 we present a Hamiltonian structure together with a Poisson bracket in section 5. Finally, we make some conclusions and outline possible generalizations.

\section{Dynamical condition}

In the present section we briefly sketch the main result presented in [四]. The Born-Infeld nonlinear electrodynamics [2] is based on the following Lagrangian (we use the HeavisideLorentz system of units with the velocity of light $c=1$ ):

$$
\mathcal{L}_{B I}:=\sqrt{-\operatorname{det}\left(b \eta_{\mu \nu}\right)}-\sqrt{-\operatorname{det}\left(b \eta_{\mu \nu}+F_{\mu \nu}\right)}=b^{2}\left(1-\sqrt{1-2 b^{-2} S-b^{-4} P^{2}}\right),
$$

where $\eta_{\mu \nu}$ denotes the Minkowski metric with the signature $(-,+,+,+)$. The standard Lorentz invariants $S$ and $P$ are defined by: $S=-\frac{1}{4} F_{\mu \nu} F^{\mu \nu}$ and $P=-\frac{1}{4} F_{\mu \nu} \tilde{F}^{\mu \nu}\left(\tilde{F}^{\mu \nu}\right.$ denotes the dual tensor). The arbitrary parameter " $b$ " has a dimension of a field strength (Born and Infeld called it the absolute field) and it measures the nonlinearity of the theory. In the limit $b \rightarrow \infty$ the Lagrangian $\mathcal{L}_{B I}$ tends to the Maxwell Lagrangian $S$.

Adding to (1) the standard electromagnetic interaction term " $j^{\mu} A_{\mu}$ " we may derive the inhomogeneous field equations

$$
\partial_{\mu} G^{\mu \nu}=-j^{\nu}
$$


where $G^{\mu \nu}:=-2 \partial \mathcal{L}_{B I} / \partial F_{\mu \nu}$. Equations (2) have formally the same form as Maxwell equations. What makes the theory effectively nonlinear are the constitutive relations, i.e. relations between inductions $(\boldsymbol{D}, \boldsymbol{B})$ and intensities $(\boldsymbol{E}, \boldsymbol{H})$ :

$$
\begin{aligned}
\boldsymbol{E}(\boldsymbol{D}, \boldsymbol{B}) & =\frac{1}{b^{2} R}\left[\left(b^{2}+\boldsymbol{B}^{2}\right) \boldsymbol{D}-(\boldsymbol{D} \boldsymbol{B}) \boldsymbol{B}\right] \\
\boldsymbol{H}(\boldsymbol{D}, \boldsymbol{B}) & =\frac{1}{b^{2} R}\left[\left(b^{2}+\boldsymbol{D}^{2}\right) \boldsymbol{B}-(\boldsymbol{D} \boldsymbol{B}) \boldsymbol{D}\right]
\end{aligned}
$$

with $R:=\sqrt{1+b^{-2}\left(\boldsymbol{D}^{2}+\boldsymbol{B}^{2}\right)+b^{-4}(\boldsymbol{D} \times \boldsymbol{B})^{2}}$.

Now, let us assume that the external current $j^{\mu}$ in (2) is produced by a point-like particle moving along the time-like trajectory $\zeta$. Due to the nonlinearity, the system (2) is very complicated to analyse. In particular, contrary to the Maxwell case, we do not know the general solution to the inhomogeneous Born-Infeld field equations. The main idea of [1] (it was proposed in the Maxwell case in [17]) was as follows: instead of solving distribution equations (2) on the entire Minkowski space-time $\mathcal{M}$ let us treat them as a boundary problem in the region $\mathcal{M}_{\zeta}:=\mathcal{M}-\{\zeta\}$, i.e. outside the trajectory. Obviously, in order to well pose the problem we have to find an appropriate boundary condition which has to be satisfied along $\zeta$, i.e. on the boundary $\mathcal{M}_{\zeta}$.

To find this boundary condition we have analysed the asymptotic behaviour of the fields in the vicinity of a charged particle. The simplest way to do it is to use the particle's rest frame. Let $r$ denotes the radial coordinate, i.e. a distance from a particle in its rest frame. Any vector field $\boldsymbol{F}=\boldsymbol{F}(\boldsymbol{r})$ may be formally expanded in the vicinity of a charge:

$$
\boldsymbol{F}(\boldsymbol{r})=\sum_{n} r^{n} \boldsymbol{F}_{(n)}
$$

where the vectors $\boldsymbol{F}_{(n)}$ do not depend on $r$. The crucial observation is that the most singular part of $\boldsymbol{D}$ field behaves as

$$
\boldsymbol{D}_{(-2)}=\frac{e \mathcal{A}}{4 \pi} \frac{\boldsymbol{r}}{r}
$$

where, due to the Gauss law, the monopole part of the $r$-independent function $\mathcal{A}$ equals 1. Note that in the Maxwell case $\mathcal{A} \equiv 1$. Using (6) it was shown [1] that:

$$
\boldsymbol{H} \sim r^{-1}, \quad \boldsymbol{E} \sim r^{0}, \quad \boldsymbol{B} \sim r .
$$

Moreover, the following theorem was proved:

Theorem 1 Any regular solution of Born-Infeld field equations with point-like external current satisfies:

$$
\boldsymbol{E}_{(1)}^{T}=\frac{b e}{4|e|}\left(3 \boldsymbol{a}-r^{-2}(\boldsymbol{a r}) \boldsymbol{r}\right)
$$

where $\boldsymbol{E}^{T}$ stands for the transversal part of $\boldsymbol{E}$ and $\boldsymbol{a}$ denotes particle's acceleration. 
Therefore, when the particle's trajectory is a priori given, the hyperbolicity of (2) implies:

Theorem 2 The mixed (initial-boundary) value problem for the Born-Infeld equations in $\mathcal{M}_{\zeta}$ with (17) playing the role of boundary condition on $\partial \mathcal{M}_{\zeta}$ has the unique solution.

The above theorem is no longer true when we consider a particle as a dynamical object. Choosing particle's position $\boldsymbol{q}$ and velocity $\boldsymbol{v}$ as the Cauchy data for the particle's dynamics let us observe that despite the fact that the time derivatives $(\dot{\boldsymbol{D}}, \dot{\boldsymbol{B}}, \dot{\boldsymbol{q}}, \dot{\boldsymbol{v}})$ of the Cauchy data are uniquely determined by the data themselves, the evolution of the composed system is not uniquely determined. Indeed, $\dot{\boldsymbol{D}}$ and $\dot{\boldsymbol{B}}$ are given by the field equations, $\dot{\boldsymbol{q}}=\boldsymbol{v}$ and $\dot{\boldsymbol{v}}$ may be calculated from (可). Nevertheless, the initial value problem is not well posed: keeping the same initial data, particle's trajectory can be modified almost at will. This is due to the fact, that now (7) plays no longer the role of boundary condition because we use it to as a dynamical equation to determine $\boldsymbol{a}$. Therefore a new boundary condition is necessary.

It was shown in [1] that this missing condition is implied by the conservation law of the total four-momentum for the "particle + field" system:

$$
\dot{p}^{\mu}(t)=0,
$$

where $p^{\mu}$ stands for the the four-momentum in any lorentzian (laboratory) frame. Due to the field equations only 3 among 4 equations (8) are independent, i.e. the conservation of three-momentum

$$
\dot{\boldsymbol{p}}(t)=0,
$$

implies the energy conservation. It was shown in [1] that (9) is equivalent to the following Newton-like equation:

$$
m a_{k}=\frac{|e| b}{3} \mathcal{A}_{k}
$$

where $\mathcal{A}_{k}$ is the dipole part of $\mathcal{A}$, i.e. $\operatorname{DP}(\mathcal{A})=: \mathcal{A}_{k} x^{k} / r$. The above equation looks formally like a standard Newton equation. However, it could not be interpreted as the Newton equation because its r.h.s. is not a priori given (it must be calculated from field equations).

To correctly interpret (10) we have to take into account (17). Now, calculating $\boldsymbol{a}$ in terms of $\boldsymbol{E}_{(1)}^{T}$ and inserting into $(\mathbb{1 0})$ we obtain the following relation between $\boldsymbol{E}_{(1)}^{T}$ and $D_{(-2)}$ :

$$
\operatorname{DP}\left(4 r_{0}^{4}\left(\boldsymbol{E}_{(1)}^{T}\right)^{r}-\lambda_{0}\left(\boldsymbol{D}_{(-2)}\right)^{r}\right)=0
$$

where $r_{0}=\sqrt{|e| / 4 \pi b}$ and $\lambda_{0}=e^{2} / 6 \pi m$. All constants (like electric charge or particle's mass) enter into the dynamical condition (11) via two characteristic scales $r_{0}$ and $\lambda_{0}$. Obviously $r_{0}$ measures the nonlinearity of the Born-Infeld theory (in the Maxwell case $\left.r_{0} \equiv 0\right)$. The second scale $\lambda_{0}$ appears in any electrodynamics of charged particles, e.g. it appears in the Lorentz-Dirac equation: $a^{\mu}=\frac{e}{m} F_{e x t}^{\mu \nu} u_{\nu}+\lambda_{0}\left(\dot{a}^{\mu}-a^{2} u^{\mu}\right)$. The main result of [1] consists in the following 
Theorem 3 Born-Infeld field equations supplemented by the dynamical condition (11) define perfectly deterministic theory, i.e. initial data for field and particle uniquely determine the entire evolution of the system.

\section{Lagrangian structure}

In this section we show that the dynamical condition (11) may be derived from the mathematically well defined variational principle. Guided by the example of Maxwell theory one could guess that such a principle should be based on the following Lagrangian:

$$
L_{\text {total }}=L_{\text {field }}+L_{\text {particle }}+L_{i n t},
$$

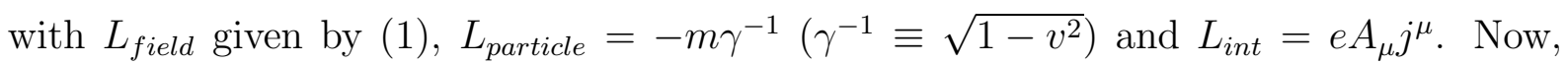
varrying $L_{\text {total }}$ with respect to $A_{\mu}$ one obviously reproduces (2). But the variation with respect to particle's trajectory leads to the standard Lorentz equation

$$
m a^{\mu}=e F^{\mu \nu} u_{\nu} .
$$

Hovewer, despite the fact that $F^{\mu \nu}$ is bounded, it is not regular at the particle's position and, therefore, the r.h.s. of (13) is not well defined. This was already our motivation to find the mathematically well defined dynamical condition (11) which replaces ill defined equations of motion (13).

Therefore, the variational principle based on (12) is not well defined. To find the correct principle consider more carefully the field dynamics with respect to an arbitrary moving observer (see [18] and [19] for a general discussion). Consider an observer moving along an arbitrary (time-like) trajectory $\zeta$. At each point $(t, \boldsymbol{q}(t)) \in \zeta$ let $\Sigma_{t}$ denotes a 3-dimensional hyperplane orthogonal to the four-velocity vector $U=\left(u^{\mu}\right)$. Choose on $\Sigma_{t}$ any system $\left(x^{i}\right)$ of cartesian coordinates, such that an observer is located at its origin. This system is not uniquely defined because on each $\Sigma_{t}$ we have still the freedom of an $O(3)$-rotation. Let $\mathbf{L}$ denotes the boost relating the laboratory time axis $\partial / \partial y^{0}$ with the co-moving observer's proper time axis $U$. Next, define the position of the $\partial / \partial x^{k}$ - axis on $\Sigma_{t}$ by transforming the corresponding $\partial / \partial y^{k}$ - axis of the laboratory frame by the same boost. It is easy to check, that one obtains:

$$
\begin{aligned}
y^{0}(t, \boldsymbol{x}) & :=t+\gamma(t) x^{l} v_{l}(t), \\
y^{k}(t, \boldsymbol{x}) & :=q^{k}(t)+\mathbf{L}_{l}^{k}(t) x^{l},
\end{aligned}
$$

where the boost

$$
\mathbf{L}_{l}^{k}:=\delta_{l}^{k}+\gamma\left(1+\gamma^{-1}\right)^{-1} v^{k} v_{l} .
$$

The flat Minkowski metric tensor has in the new coordinates $\left(x^{0}=t, x^{k}\right)$ the following form: $g_{k l}=\delta_{k l}$, whereas the lapse function $N$ and the purely rotational shift vector $\boldsymbol{N}$ read:

$$
\begin{aligned}
N & =\frac{1}{\sqrt{-g^{00}}}=\gamma^{-1}(1+\boldsymbol{a x}), \\
N_{m} & =g_{0 m}=\gamma^{-1}(\boldsymbol{\omega} \times \boldsymbol{x})_{m},
\end{aligned}
$$


where $\boldsymbol{a}$ is the observer's acceleration vector in the co-moving frame

$$
a^{i}=\gamma^{2} \mathbf{L}_{l}^{i} \dot{v}^{l}
$$

and

$$
\boldsymbol{\omega}=\gamma^{2}\left(1+\gamma^{-1}\right)^{-1} \boldsymbol{v} \times \dot{\boldsymbol{v}} .
$$

The field equations have in the co-moving frame the following form (cf. a general discussion in [20]):

$$
\begin{aligned}
\dot{\boldsymbol{D}} & =\gamma^{-1}[\nabla \times(N \boldsymbol{H})+(\boldsymbol{N} \cdot \nabla) \boldsymbol{D}-(\boldsymbol{D} \cdot \nabla) \boldsymbol{N}], \\
\dot{\boldsymbol{B}} & =\gamma^{-1}[-\nabla \times(N \boldsymbol{E})+(\boldsymbol{N} \cdot \nabla) \boldsymbol{B}-(\boldsymbol{B} \cdot \nabla) \boldsymbol{N}] .
\end{aligned}
$$

In [1] we used a simplified (so called Fermi-Walker) frame in which the shift vector $\boldsymbol{N}=0$. However, such a frame is nonlocal in time and, therefore, one can not use it to define the Hamiltonian structure in a consistent way. Observe, that the field evolution given by (19) is a superposition of the following three transformations:

- time-translation in the direction of the observer's four-velocity $U$,

- boost in the direction of the observer's acceleration $\boldsymbol{a}$,

- purely spatial $O(3)$-rotation around the vector $\boldsymbol{\omega}$.

It is, therefore, obvious that this evolution is generated by the following generator:

$$
H_{\text {field }}:=-\gamma^{-1}\left(\mathcal{P}^{0}+\boldsymbol{a} \mathcal{R}-\boldsymbol{\omega} \mathcal{S}\right),
$$

where $\mathcal{P}^{0}$ is the field energy, $\mathcal{R}$ is the static moment and $\mathcal{S}$ denotes the angular momentum. These quantities are defined in the observer's rest frame via a symmetric energymomentum tensor:

$$
T^{\mu}{ }_{\nu}=\delta^{\mu}{ }_{\nu} \mathcal{L}_{B I}-\frac{\partial \mathcal{L}_{B I}}{\partial S} F_{\lambda}^{\mu} F^{\lambda}{ }_{\nu}-\frac{\partial \mathcal{L}_{B I}}{\partial P} F_{\lambda}^{\mu} \tilde{F}_{\nu}^{\lambda} .
$$

The relativistic factor $\gamma^{-1}$ corresponds to the fact that we use not the proper time along the observer's trajectory $\zeta$ but the laboratory time. The "-" is choosen for the future convenience. We stress that $H_{\text {field }}$ plays the role of a Hamiltonian (with negative sign) for any relativistic lagrangian field theory. In the case of electrodynamics (Maxwell or general nonlinear theory) the corresponding Hamilton equations are given by (19).

Now, let us add to this picture a charged particle by replacing the field energy $\mathcal{P}^{0}$ by a total "particle + field" energy $\mathcal{H}=m+\mathcal{P}^{0}$. Obviously, the particle's static moment and angular momentum vanish in its rest frame. Define the new generator

$$
L_{H}:=-\gamma^{-1}(\mathcal{H}+\boldsymbol{a} \mathcal{R}-\boldsymbol{\omega S}) .
$$

It turns out that the above generator contains all information about the particle's dynamics. This is due to the following 
Theorem 4 The generator $L_{H}$ defines the 2-nd order particle's Lagrangian, i.e. varrying it with respect to the particle's trajectory one recovers the dynamical condition (11).

Proof: the Euler-Lagrange equations for a 2-nd order Lagrangian read:

$$
\dot{\boldsymbol{p}}=\frac{\partial L_{H}}{\partial \boldsymbol{q}},
$$

where the momentum $\boldsymbol{p}$ canonically conjugated to the particle's position $\boldsymbol{q}$ is defined by

$$
\boldsymbol{p}:=\frac{\partial L_{H}}{\partial \boldsymbol{v}}-\frac{d}{d t}\left(\frac{\partial L_{H}}{\partial \dot{\boldsymbol{v}}}\right)
$$

(see 21] for a general discussion of higher order Lagrangians. A review of a 2-nd order case may be found in [18] and [19]). To calculate $\boldsymbol{p}$ one needs time derivatives of $\boldsymbol{\mathcal { R }}$ and $\mathcal{S}$. Using field equations (19) and the asymtotic conditions presented in the previous section one gets:

$$
\begin{aligned}
\dot{\mathcal{R}} & =\gamma^{-1}(\mathcal{P}-\boldsymbol{a} \times \mathcal{S}-\boldsymbol{\omega} \times \mathcal{R}) \\
\dot{\mathcal{S}} & =\gamma^{-1}(\boldsymbol{a} \times \mathcal{R}-\boldsymbol{\omega} \times \mathcal{S})
\end{aligned}
$$

where $\mathcal{P}$ denotes the field momentum in the co-moving frame. Therefore, one obtains the following formula

$$
p_{k}=\gamma v_{k} \mathcal{H}+\mathbf{L}^{l}{ }_{k} \mathcal{P}_{l}+\mathbf{A}^{l}{ }_{k} \mathcal{R}_{l}+\mathbf{B}^{l}{ }_{k} \mathcal{S}_{l}
$$

with

$$
\begin{aligned}
& \mathbf{A}_{k}^{l}=\frac{d}{d t}\left(\gamma^{-1} \frac{\partial a^{l}}{\partial \dot{v}^{k}}\right)-\frac{\partial\left(\gamma^{-1} a^{l}\right)}{\partial v^{k}}-\gamma^{-2} \epsilon_{m}{ }^{i l}\left(\frac{\partial a^{m}}{\partial \dot{v}^{k}} \omega_{i}+\frac{\partial \omega^{m}}{\partial \dot{v}^{k}} a_{i}\right), \\
& \mathbf{B}_{k}^{l}=-\frac{d}{d t}\left(\gamma^{-1} \frac{\partial \omega^{l}}{\partial \dot{v}^{k}}\right)+\frac{\partial\left(\gamma^{-1} \omega^{l}\right)}{\partial v^{k}}+\gamma^{-2} \epsilon_{m}{ }^{i l}\left(\frac{\partial a^{m}}{\partial \dot{v}^{k}} a_{i}+\frac{\partial \omega^{m}}{\partial \dot{v}^{k}} \omega_{i}\right) .
\end{aligned}
$$

After straightforward (but tedious) algebra one finds: $\mathbf{A}^{l}{ }_{k}=\mathbf{B}^{l}{ }_{k}=0$ and finally

$$
p_{k}=\gamma v_{k} \mathcal{H}+\mathbf{L}^{l}{ }_{k} \mathcal{P}_{l} .
$$

But (27) is nothing else than a total "particle + field" momentum in the laboratory frame. Therefore, the Euler-Lagrange equations (22) reduce to the conservation law of the total momentum (since the r.h.s. of (22) vanishes in our case) and it was proved in [1] that it is equivalent to the dynamical condition (111). This ends the proof.

\section{Hamiltonian}

To find the corresponding Hamiltonian structure of the above theory one has to perform the Legendre transformation to $L_{H}$. Let $\boldsymbol{\pi}$ denotes the momentum canonically conjugated to particle's velocity $\boldsymbol{v}$, i.e.

$$
\boldsymbol{\pi}:=\frac{\partial L_{H}}{\partial \dot{\boldsymbol{v}}} .
$$


Observe, that due to the fact that $L_{H}$ is linear in $\dot{\boldsymbol{v}}$ (see (17) and (18)) it is impossible to invert (28) (i.e. to calculate $\boldsymbol{v}$ in terms of $\boldsymbol{\pi}$ ) and, therefore, the Legendre transformation is singular. It means that in the Hamiltonian framework the phase space of our system

$$
\mathcal{P}=(\boldsymbol{q}, \boldsymbol{p}, \boldsymbol{v}, \boldsymbol{\pi} ; \boldsymbol{D}, \boldsymbol{B})
$$

is subjected to some constraints. To find these constraints let us apply the standard Dirac-Bergmann procedure [22] (see also [23]). The phase space $\mathcal{P}$ is endowed with the following canonical Poisson bracket:

$$
\{\mathcal{F}, \mathcal{G}\}=\frac{\partial \mathcal{F}}{\partial \boldsymbol{q}} \cdot \frac{\partial \mathcal{G}}{\partial \boldsymbol{p}}+\frac{\partial \mathcal{F}}{\partial \boldsymbol{v}} \cdot \frac{\partial \mathcal{G}}{\partial \boldsymbol{\pi}}+\int_{\Sigma} \frac{\delta \mathcal{F}}{\delta \boldsymbol{D}} \cdot \nabla \times \frac{\delta \mathcal{G}}{\delta \boldsymbol{B}} d^{3} x-(\mathcal{F} \rightleftharpoons \mathcal{G}) .
$$

Now, the (unreduced) Hamiltonian on $\mathcal{P}$ is defined by:

$$
H(\boldsymbol{q}, \boldsymbol{p}, \boldsymbol{v}, \boldsymbol{\pi} ; \boldsymbol{D}, \boldsymbol{B})=\boldsymbol{p} \boldsymbol{v}+\boldsymbol{\pi} \dot{\boldsymbol{v}}-L_{H} .
$$

Therefore, the primary and secondary constraints read:

$$
\begin{aligned}
\phi_{k}^{(1)} & :=\pi_{k}-\frac{\partial L_{H}}{\partial \dot{v}^{k}} \approx 0 \\
\phi_{k}^{(2)} & :=\left\{\phi_{k}^{(1)}, H\right\}=-p_{k}+\gamma v_{k} \mathcal{H}+\mathbf{L}^{l}{ }_{k} \mathcal{P}_{l} \approx 0
\end{aligned}
$$

where the symbol " $\approx$ " refers to the "weak equality". Observe, that the secondary constraints $\phi_{k}^{(2)}=0$ reproduce (27). Using (31) and (32) we get

$$
H=\boldsymbol{p} \boldsymbol{v}+\gamma^{-1} \mathcal{H}+\dot{v}^{k} \phi_{k}^{(1)}
$$

and $\dot{v}^{k}$ plays now the role of Lagrange multiplier. Let us continue with the DiracBergmann procedure and look for the tetriary constraints:

$$
\phi_{k}^{(3)}:=\left\{\phi_{k}^{(2)}, H\right\} \approx 0,
$$

with $H$ given by (33). One may show (following the calculations in [1]) that (34) implies

$$
\dot{v}^{k}=\gamma^{-2} \frac{|e| b}{3 m}\left(\mathbf{L}^{-1}\right)^{k}{ }_{l} \mathcal{A}^{l}
$$

which is equivalent to (10). Together with (7) it implies our dynamical condition (11). Therefore, the consistency of the theory requires that the dynamical condition has to be already present in the definition of the physical phase space. This way the tetriary constraints are identically satisfied and the constraint algorithm stops at this point. Observe, that the Lagrange multiplier $\boldsymbol{v}$ in (33) is, therefore, already known which means that the constraints $\phi_{k}^{(1)}$ and $\phi_{k}^{(2)}$ are of the second class (see the next section).

Now, on the reduced phase space, i.e. $\mathcal{P}$ subjected to (31) and (32) (and to the dynamical condition (11)) the reduced Hamiltonian

$$
H^{*}(\boldsymbol{q}, \boldsymbol{v} ; \boldsymbol{D}, \boldsymbol{B})=\gamma(\mathcal{H}+\boldsymbol{v} \mathcal{P})
$$


equals to the total energy of the composed system in the laboratory frame. In deriving (36) we chose as independent variables (in the particle's sector) particle's position $\boldsymbol{q}$ and velocity $\boldsymbol{v}$ (this choice is strongly sugested by the form of constraints equations). Obviously, this parameterization is not unique. For example we could take as independent variables $\boldsymbol{q}$ and $\boldsymbol{p}$ as well. One could show (see [19]) that

$$
\boldsymbol{v}=\frac{[\boldsymbol{p}(\boldsymbol{p}-\mathcal{P})]\left(\sqrt{\mathcal{H}^{2}+\boldsymbol{p}^{2}-\mathcal{P}^{2}}-\mathcal{H}\right)}{[\boldsymbol{p}(\boldsymbol{p}-\mathcal{P})]^{2}+\mathcal{H}^{2}(\boldsymbol{p}-\mathcal{P})^{2}}(\boldsymbol{p}-\mathcal{P})
$$

and, therefore

$$
H^{*}(\boldsymbol{q}, \boldsymbol{p} ; \boldsymbol{D}, \boldsymbol{B})=\sqrt{\mathcal{H}^{2}+\boldsymbol{p}^{2}-\mathcal{P}^{\mathbf{2}}} .
$$

Obviously, numerically $H^{*}(\boldsymbol{q}, \boldsymbol{v} ; \boldsymbol{D}, \boldsymbol{B})=H^{*}(\boldsymbol{q}, \boldsymbol{p} ; \boldsymbol{D}, \boldsymbol{B})$. Observe, that for a free particle, i.e. $e=0, \mathcal{H}=m$ and $\mathcal{P}=0$, the complicated formula (37) reduces to the relativistic relation between particle's velocity and momentum:

$$
\boldsymbol{v}=\frac{\boldsymbol{p}}{\sqrt{m^{2}+\boldsymbol{p}^{2}}}
$$

and formula (38) reproduces the relativistic particle's energy: $E(\boldsymbol{p})=\sqrt{m^{2}+\boldsymbol{p}^{2}}$.

\section{Poisson bracket}

In this section we reduce the Poisson bracket (29) (defined on $\mathcal{P}$ ) on the reduced phase space

$$
\mathcal{P}^{*}=(\boldsymbol{q}, \boldsymbol{v} ; \boldsymbol{D}, \boldsymbol{B}) .
$$

This is possible because, as one can easily check, the constraints (31) and (32) are of the second class:

$$
\begin{aligned}
\left\{\phi_{k}^{(1)}, \phi_{l}^{(1)}\right\} & =0 \\
\left\{\phi_{k}^{(1)}, \phi_{l}^{(2)}\right\} & =-\gamma m\left(g_{k l}+\gamma^{2} v_{k} v_{l}\right) \\
\left\{\phi_{k}^{(2)}, \phi_{l}^{(2)}\right\} & =\frac{\gamma|e| b}{3}\left(v_{k} \mathcal{A}_{l}-v_{l} \mathcal{A}_{k}\right) .
\end{aligned}
$$

Therefore, due to the rules of Dirac-Bergmann procedure 22 we obtain the following formula for the reduced Poisson (or Dirac) bracket on $\mathcal{P}^{*}$ :

$$
\begin{aligned}
\{\mathcal{F}, \mathcal{G}\}^{*} & =\{\mathcal{F}, \mathcal{G}\}+\mathbf{X}^{k l}\left(\left\{\mathcal{F}, \phi_{k}^{(2)}\right\}\left\{\phi_{l}^{(1)}, \mathcal{G}\right\}-\left\{\mathcal{F}, \phi_{k}^{(1)}\right\}\left\{\phi_{l}^{(2)}, \mathcal{G}\right\}\right) \\
& -\mathbf{Y}^{k l}\left\{\mathcal{F}, \phi_{k}^{(1)}\right\}\left\{\phi_{l}^{(1)}, \mathcal{G}\right\}
\end{aligned}
$$

where

$$
\begin{aligned}
\mathbf{X}^{k l} & =\frac{g^{k l}-v^{k} v^{l}}{\gamma m} \\
\mathbf{Y}^{k l} & =\frac{|e| b}{3 m^{2} \gamma^{3}}\left(v^{k} \mathcal{A}^{l}-v^{l} \mathcal{A}^{k}\right)
\end{aligned}
$$


In particular one easily shows that in the "particle's sector":

$$
\begin{aligned}
& \left\{q^{k}, q^{l}\right\}^{*}=0, \\
& \left\{q^{k}, v^{l}\right\}^{*}=\mathbf{X}^{k l}, \\
& \left\{v^{k}, v^{l}\right\}^{*}=\mathbf{Y}^{k l} .
\end{aligned}
$$

Others "commutation relations" between variables parameterizing $\mathcal{P}^{*}$ may be easily obtained from (40).

Observe, that using (40) we are able to reproduce the dynamics of our theory:

$$
\begin{aligned}
& \dot{q}^{k}=\left\{q^{k}, H^{*}\right\}^{*}=v^{k} \\
& \dot{v}^{k}=\left\{v^{k}, H^{*}\right\}^{*}=\gamma^{-2} \frac{|e| b}{3 m}\left(\mathbf{L}^{-1}\right)^{k}{ }_{l} \mathcal{A}^{l},
\end{aligned}
$$

and in the "field sector" one easily finds that

$$
\begin{aligned}
\dot{D}^{k} & =\left\{D^{k}, H^{*}\right\}^{*} \\
\dot{B}^{k} & =\left\{B^{k}, H^{*}\right\}^{*}
\end{aligned}
$$

are equivalent to (19) where $\dot{\boldsymbol{v}}$ is given now by (47). Observe, that (47) is nothing more than an identity because the dynamical condition (which is equivalent to (47) together with (7)) is already present in the definition of $\mathcal{P}^{*}$. This way we have proved

Theorem 5 The triple $\left(\mathcal{P}^{*}, H^{*},\{,\}^{*}\right)$ defines mathematically consistent canonical structure of a point-like charge particle interacting with nonlinear Born-Infeld electromagnetism.

As a simple implication one can prove

Theorem 6 Laboratory-frame Lorentz generators:

$$
\begin{aligned}
p^{0} & =\gamma\left(\mathcal{H}+v^{l} \mathcal{P}_{l}\right) \\
p_{k} & =\gamma v_{k} \mathcal{H}+\mathbf{L}^{l}{ }_{k} \mathcal{P}_{l}, \\
r_{k} & =\gamma^{3}\left(\mathbf{L}^{-1}\right)^{l}{ }_{k} \mathcal{R}_{l}+\gamma \epsilon_{k l m} v^{l} \mathcal{S}^{m}+q_{k} p^{0}, \\
s_{k} & =\gamma^{3}\left(\mathbf{L}^{-1}\right)^{l}{ }_{k} \mathcal{S}_{l}-\gamma \epsilon_{k l m} v^{l} \mathcal{R}^{m}+\epsilon_{k l m} q^{l} p^{m}
\end{aligned}
$$

form with respect to $\{,\}^{*}$ the Poincaré algebra.

This shows that the canonical structure of our theory is perfectly consistent with the relativistic invariance.

\section{Concluding remarks}

Finally, let us make the following remarks:

1. A formalism applied in the present paper is perfectly gauge-invariant. There is no need to use a gauge potential to couple a particle to the field. 
2. The 2-nd order particle's Lagrangian $L_{H}$ can not be written in the form as in formula (12). In particular there is no "interaction term" in $L_{H}$. All information about the interaction between a particle and fields is contained in the asymptotic conditions for the electromagnetic field in the vicinity of particle's trajectory. Observe that $L_{H}$ serves as a Lagrangian for a particle's dynamics and a Hamiltonian for the field dynamics. Therefore, it is a nontrivial example of a so called Routhian function known from analytical mechanics.

3. A total four-momentum $p^{\mu}$ of the composed "particle + field" system lies always (as it should) in the future lightcone (this obvious property does not hold in the Maxwell case [17]).

4. The remarkable feature of the reduced Poisson bracket is that it is analytical in the point $e=0$. In our opinion this property is crucial for the construction of the consistent electrodynamics of point-like objects. In the Maxwell case it is well known that all unphysical solutions to the Lorentz-Dirac equation are nonanalytical at $e=0$ 10. It turns out [19] that this nonanalyticity is also present in the Hamiltonian framework where we do not have any equations of motion (there is an analog of the dynamical condition). Namely, the Poisson bracket is nonanalytical in $e=0$. Therefore, the analyticity of the canonical structure is a necessary condition for the consistency of the theory. This point will be carefully discussed in the next paper.

5. It is clear from (39) that in the case of purely electromagnetical particle (i.e. when

the effective mass $m=0)$ the constraints $\phi_{k}^{(1)}$ and $\phi_{k}^{(2)}$ are no longer of a second class $-\phi_{k}^{(1)}$ are first class. Therefore, they give rise to a certain gauge freedom and the reduced phase space $\mathcal{P}^{*}=(\boldsymbol{q}, \boldsymbol{v} ; \boldsymbol{D}, \boldsymbol{B})$ is not a proper space of states in this case, i.e. the dynamics of our system can not be consistently reduced on $\mathcal{P}^{*}$. This means that the data $(\boldsymbol{q}, \boldsymbol{v} ; \boldsymbol{D}, \boldsymbol{B})$ does not determine the evolution uniquely (there is a gauge freedom). The observation that the purely electromagnetical particles do not have consistent Hamiltonian formulation was made long ago by Pryce (see section 8 of [16]).

6. Finally, observe that variables $(\boldsymbol{q}, \boldsymbol{v} ; \boldsymbol{D}, \boldsymbol{B})$ are highly noncanonical with respect to the reduced Poisson bracket. It would be interesting to find a canonical set.

The results obtained in the present paper could be generalized

1. to include many charged particles,

2. to include Born-Infeld dyons, i.e. particles posessing both electric and magnetic charges.

Work on these points is in progress.

\section{Acknowledgements}

I would like to thank Prof. J. Kijowski for many discussions about the canonical formalism for classical field theories, Prof. I. Białynicki-Birula for pointing out the reference [16] and Prof. H. Römer for his interest in this work. The financial support from the Alexander von Humboldt Stiftung is gratefully acknowledged. 


\section{References}

[1] Chruściński D 1997 Point Charge in the Born-Infeld Electrodynamics Preprint hepth/9712161 (to appear in Phys. Lett. A.)

[2] Born M 1934 Proc. Roy. Soc. A 143 410;

Born M and Infeld L 1934 Proc. Roy. Soc. A 144425.

[3] Białynicki-Birula I and Białynicka-Birula 1975 Quantum Electrodynamics (Oxford: Pergamon);

Białynicki-Birula I 1983 Nonlinear Electrodynamics: Variations on a Theme of Born and Infeld in Quantum Theory of Particles and Fields Eds. B. Jancewicz and J. Lukierski, World Scientific, 31-48.

[4] Gibbons G W 1997 Born-Infeld particles and Dirichlet p-branes Preprint hepth/9709027.

[5] Dirac P A M 1938 Proc. Roy. Soc. A 167148.

[6] Bachas C 1997 (Half) a lecture on D-branes Preprint hep-th/9701019;

Fradkin E S and Tseytlin A A 1985 Phys. Lett. B 163 123;

Perry M and Schwarz J H 1996 Interacting chiral gauge fields in six dimensions and Born-Infeld theory Preprint hep-th/9611065;

[7] Olive D 1997 Nucl. Phys. (Proc. Suppl.) B 5843.

[8] Gibbons G W and Rasheed D A 1995 Nucl. Phys. B 454 185;

Gibbons G W and Rasheed D A 1996 Phys. Lett. B 365 46;

Gaillard M K and Zumino B 1997 Self-duality in nonlinear electromagnetism Preprint hep-th/9705226;

Bengtsson I 1996 Manifest duality in Born-Infeld theory Preprint hep-th/9612174.

[9] Schrödinger E 1935 Proc. Roy. Soc. A 150466.

[10] Rohrlich F 1965 Classical Charged Particles Addison-Wesley Reading.

[11] Heisenberg W and Euler H 1936 Z. Phys. 98 714;

Euler H and Kockel B 1935 Naturwissenschaften 23246.

[12] Hagiwara T 1981 Nucl. Phys. B 189135.

[13] Hagiwara T 1981 J. Phys. A: Math. Gen. 143059.

[14] Plebański J 1968 Lectures on Non-linear Electrodynamics (Copenhagen: Nordita). 
[15] Chruściński D and Kijowski J 1997 C. R. Acad. Sci. Paris 324 Serié IIb 435;

Chruściński D and Kijowski J 1998 J. Phys. A: Math. Gen. 31269.

[16] Pryce H L M 1936 Proc. Roy. Soc. A 155597.

[17] Kijowski J 1994 Gen. Rel. Grav. 26 167;

Kijowski J 1994 Acta Phys. Polon. A 85771.

[18] Kijowski J and Chruściński D 1995 Gen. Rel. Grav. 27267.

[19] Chruściński D 1997 Hamiltonian Structure for Classical Electrodynamics of a Point Particle Preprint physics/9712014 (to appear in Rep. Math. Phys.).

[20] Misner C Thorne K S and Wheeler J A 1973 Gravitation (W. H. Freeman and Co.: San Francisco).

[21] Gitman D M and Tyutin I V 1990 Quantization of Fields with Constraints (SpringerVerlag: Berlin).

[22] Dirac P A M 1950 Can. J. Math. 2 129;

Dirac P A M 1964 Lectures on quantum mechanics Belfer Graduate School of Science Monograph Series;

Bergmann P 1961 Rev. Mod. Phys. 33510.

[23] Hanson A J Regge T and Teitelboim C 1976 Constrained Hamiltonian Systems Rome: Accad. Naz. de Lincei.;

Sundermeyer K 1982 Constrained Dynamics Lecture Notes in Physics 169 (SpringerVerlag: Berlin);

Henneaux M and Teitelboim C 1992 Quantization of Gauge Systems (Princeton University Press: Princeton). 